University of Wollongong

Research Online

Faculty of Arts, Social Sciences and Humanities

- Papers

Faculty of Arts, Social Sciences \& Humanities

2020

Can Parental Engagement in Social Media Enhance Outcomes of an Online Healthy Lifestyle Program for Preschool-Aged Children?

Megan L. Hammersley

University of Wollongong, mhammers@uow.edu.au

Anthony D. Okely

University of Wollongong, tokely@uow.edu.au

Marijka Batterham

University of Wollongong, marijka@uow.edu.au

Rachel A. Jones

University of Wollongong, rachelj@uow.edu.au

Follow this and additional works at: https://ro.uow.edu.au/asshpapers

Research Online is the open access institutional repository for the University of Wollongong. For further information contact the UOW Library: research-pubs@uow.edu.au 


\title{
Can Parental Engagement in Social Media Enhance Outcomes of an Online Healthy Lifestyle Program for Preschool-Aged Children?
}

\author{
Abstract \\ This study explored parental engagement, child BMI and secondary outcomes from the social media \\ component of an online healthy lifestyle program for parents of preschool-aged children. Intervention \\ group participants received access to an online program and Facebook group. Data were collected at \\ baseline and 3- and 6-months follow-up. Facebook usage data on comments and posts were used to \\ determine total active engagement. There was a high level of Facebook group membership and most \\ parents actively engaged at least once. Although there were varying levels of engagement between \\ modules and cohorts, it was modest overall. User acceptability of the Facebook group was lower than \\ expected. Children of parents in the intervention who engaged more in the Facebook group (by posting \\ and commenting) demonstrated greater sleep duration over time (estimate $1.79,95 \% \mathrm{Cl} 0.42$ to $3.17, \mathrm{p}$ \\ $=.01$ ) Children of parents who engaged more in the Facebook group also participated in less moderate- to \\ vigorous-intensity physical activity (estimate $-0.14,95 \% \mathrm{Cl}-0.26$ to $-0.01, \mathrm{p}=.03$ ). This study is one of \\ the first parent-focussed healthy lifestyle interventions to include a social media component. Further \\ research is recommended with larger sample sizes and longer duration to further explore the potential of \\ social media in childhood obesity interventions.

\section{Publication Details} \\ Hammersley, M. L., Okely, A. D., Batterham, M. J. \& Jones, R. A. (2020). Can Parental Engagement in \\ Social Media Enhance Outcomes of an Online Healthy Lifestyle Program for Preschool-Aged Children?. \\ Health Communication, 35 (9), 1162-1171.
}




\section{Can parental engagement in social media enhance outcomes of an online healthy lifestyle program for preschool-aged children?}

Dr Megan L Hammersley, Early Start, Faculty of Social Sciences, University of Wollongong.

Professor Anthony D Okely, Early Start, Faculty of Social Sciences, University of

Wollongong.

Associate Professor Marijka J Batterham, Statistical Consulting Service, National Institute for Applied Statistics Research Australia, School of Mathematics and Applied Statistics, University of Wollongong.

Dr Rachel A Jones, Early Start, Faculty of Social Sciences, University of Wollongong.

Funding Source: This research has been conducted with the support of the Australian Government Research Training Program Scholarship. The study was also supported by funding from Australian Health Management. The funding body was not involved in the design, data collection, analysis, interpretation or writing.

Acknowledgements: This research has been conducted with the support of the Australian Government Research Training Program Scholarship. The study was also supported by funding from Australian Health Management. The funding body was not involved in the design, data collection, analysis, interpretation or writing. 


\begin{abstract}
This study explored parental engagement, child BMI and secondary outcomes from the social media component of an online healthy lifestyle program for parents of preschoolaged children. Intervention group participants received access to an online program and Facebook group. Data were collected at baseline, 3- and 6-months. Facebook usage data on comments and posts were used to determine total active engagement. There was a high level of Facebook group membership and most parents actively engaged at least once. Although there were varying levels of engagement between modules and cohorts, it was modest overall. User acceptability of the Facebook group was lower than expected. Children of parents in the intervention who engaged more in the Facebook group (by posting and commenting) demonstrated greater sleep duration over time (estimate $1.79,95 \%$ CI 0.42 to $3.17, \mathrm{p}=0.01)$ Children of parents who engaged more in the Facebook group also participated in less moderate- to vigorous-intensity physical activity (estimate $-0.14,95 \% \mathrm{CI}-0.26$ to $0.01, p=0.03$ ). This study is one of the first parent-focussed healthy lifestyle interventions to include a social media component. Further research is recommended with larger sample sizes and longer duration to further explore the potential of social media in childhood obesity interventions.
\end{abstract}


Effective community-based health behavior change interventions are key to improving obesity-related behaviors in childhood (World Health Organization, 2012). Such interventions to date have largely concentrated on improving physical activity and eating behaviors. There is also increasing evidence regarding the importance of , reducing sedentary behavior (Okely \& Jones, 2011; Okely et al., 2012) and maintaining adequate sleep patterns (Miller, Kruisbrink, Wallace, Ji, \& Cappuccio, 2018). During early childhood, parental influence and role-modelling play a key part in the development of healthy behaviors, making positive influence of parents at this stage vitally important (Golley, Hendrie, Slater, \& Corsini, 2011; Natale et al., 2014).

Parent-focused interventions which have embraced eHealth technologies have shown promise in improving childhood obesity-related behaviors such as dietary intake and physical activity (Chen, Weiss, Heyman, Cooper \& Lustig, 2011; Hammersley, Okely, Batterham \& Jones, 2019; Williamson et al., 2005). Additionally, health behavior change interventions that incorporate interactive social media have shown potential (Thackeray, Neiger, Hanson, McKenzie, 2008). Behaviour change interventions that include a social media aspect are potentially more attractive because this communication and interaction method is widely utilised by different age groups (Welch et al., 2018), it is generally a cost effective addition to interventions (Moorhead, Hazlett, Harrison, Carroll, Irwin \& Hoving, 2013) and often results in greater retention (De Bruyn \& Lilien, 2008).

There is a high rate of social media usage among parents (Duggan, Lenhart, Lampe \& Ellison, 2015) who use these sites to keep in touch, obtain and share information, seek and provide support, and reduce social isolation (Strange, Fisher, Howat \& Wood, 2018). Social media can be a convenient, easy to access and time-efficient alternative to group social support (O'Kane et al., 2018; Haslam, Tee \& Baker, 2017) at a life stage life when there is 
often limited time and opportunity to connect face-to-face due to child caring responsibilities, sleep routines and other considerations (Strange et al., 2018).

Parents of young children also access social media sites to help support their role as a parent (Doub, Small \& Birch, 2016; Duggan et al., 2015), through seeking parenting information, asking specific parenting questions, or obtaining social and emotional support on parenting issues (Duggan et al., 2015). Over $80 \%$ of parents with internet access use social media sites (Duggan et al., 2015) and these sites offer thousands of parenting support groups (Niela-Vilén, Axelin, Salantera \& Melender, 2014). Social media options are extensive; however at the current time, Facebook is the most popular, with over 2 billion users worldwide (Smart Insights, 2018). In Australia, 94\% of social media users have a Facebook account (Sensis, 2017). Facebook is the most popular social media site among parents and the majority of parent users log on at least once a day (Duggan et al., 2015).

A recent review indicated that there is limited evidence of the efficacy of social media interventions which focus on child health; however these interventions treated children directly (Hamm et al., 2014). A small number of interventions incorporating social media have targeted parents, but few have focused on childhood obesity-related behaviors (Downing, Campbell \& van der Plight, 2017; Fiks et al., 2017, Gruver et al., 2016; Ruotsalainen, Kyngas, Tammelin, Hekkinen \& Kaariainen, 2015; Ling et al., 2018, Swindle, Ward \& Whiteside-Mansell, 2018). These studies have included RCTs (Downing et al., 2017; Fiks et al., 2017; Ruotsalainen et al., 2015;) and feasibility studies (Gruver et al., 2016; Ling et al., 2018; Swindle et al., 2018) in infant, preschool and adolescent age groups.

While some interventions have not reported using a theoretical basis (Downing et al., 2017), most studies which have used a theory have adopted Social Cognitive Theory (SCT) or Social Learning Theory to guide the development of the intervention, using social media 
as a tool for participants to share information and experiences, gain social support and learn from observing each other (Fiks et al., 2017; Gruver et al., 2016; Ling et al., 2018).

Most studies have used social media as a component of a broader intervention (Downing et al., 2017; Ling et al., 2018; Ruotsalainen et al., 2015; Swindle et al., 2018), with the exception of Fiks et al (2017) and Gruver et al (2016), who used Facebook as the main intervention. Feasibility studies have generally reported good engagement and user acceptance. RCTs have reported a high level of user acceptance and varying levels of engagement and two studies, both in infant age groups, reported positive dietary intake outcomes (Downing et al., 2017; Fiks et al., 2017). Two feasibility studies have been conducted in the preschool-age group (Ling et al., 2018; Swindle et al., 2018), with both of these studies reporting that social media is an acceptable platform to use as a component in childhood obesity prevention and healthy lifestyle interventions involving parents and therefore further research is warranted.

The Time2bHealthy study was a RCT of an online healthy lifestyle program for parents of 2-5 year old children, which included a Facebook component. The main outcomes analyses found that children in the intervention group reduced their frequency of discretionary food intake, and parents improved their attitudes and practices regarding pressuring their child to eat and their self-efficacy in regard to nutrition compared to those in the comparison group (Hammersley et al., 2019). This current paper aimed to determine if engagement in the Facebook component of the Time2bHealthy intervention influenced child and parent-related outcomes. It was hypothesized that participants who displayed greater levels of active engagement in the social media component of the program would achieve superior outcomes in change in child BMI, dietary intake, physical activity, sleep and child feeding compared to participants who had a lower level of engagement. 


\section{Methods}

\section{Study design}

The Time2bHealthy RCT was conducted between January 2016 and December 2017 (Hammersley et al., 2019). The trial was approved by the University of Wollongong Human Research Ethics Committee (HE15/354) and registered with the Australian and New Zealand Clinical Trials Registry (ACTRN12616000119493). Participants provided informed consent prior to participating and were assigned to one of six cohorts on a rolling basis. The Time2bHealthy online program was 11-weeks in duration with a post-intervention and 3month follow-up.

\section{Participant recruitment and eligibility}

Interested parents were screened over the phone or via email to determine provisional eligibility. Children were eligible if they were 2-5 years of age and their BMI was at or above the WHO $50^{\text {th }}$ percentile for their age and sex, aligning with some similar eHealth studies focused on childhood obesity prevention and obesity related behaviors (Baranowski et al., 2003; Chen et al., 2011). Eligibility was confirmed when height and weight were measured at baseline. Exclusion criteria included medical conditions or medications which affected weight or age-appropriate play. Parents were excluded if they did not have a Facebook account and were not willing to open one. Recruitment areas included the Illawarra region of New South Wales and surrounding areas and Melbourne, Victoria in Australia. The methods used have been described in detail elsewhere (Hammersley, Jones \& Okely, 2017).

\section{Time2bHealthy intervention}

The Time2bHealthy intervention has been described elsewhere (Hammersley et al., 2017). Briefly, Time2bHealthy is an 11-week online healthy lifestyle program for parents of preschool-aged children which is aligned to SCT (Bandura, 1989). Module topics include 
healthy eating (x2), physical activity, screen-time, and sleep, with each module consisting of text, videos, activities and goal-setting components. Each module took approximately 30 minutes to complete. Participants received weekly emails and feedback from the facilitator (a dietitian) on the goal-setting component. Post-program, participants received fortnightly emails until 6-month follow-up to recap on key information.

\section{Facebook component}

Intervention participants from each cohort were asked to join a closed 'secret' Facebook group, allowing the opportunity to connect with others, gain a greater understanding of program content, pose questions to others, and share ideas and experiences.

As previously described (Hammersley et al., 2017), the overall intervention, including the Facebook component was developed as a result of a backwards intervention mapping exercise where intervention activities were aligned to SCT, and the target healthy lifestyle behaviors. SCT proposes that individuals learn from their own experiences and observing experiences of others, and suggests that personal, environmental and behavioral factors interact to influence behavior. Personal influence refers to an individual's self-efficacy to perform a behavior, environmental influence refers to the physical and societal environment, and behavioral influence refers to an individual's response once a behavior has been performed (Bandura, 1989). The broader Time2bHealthy intervention embodied SCT constructs such as self-efficacy, goal-setting, reducing barriers, self-monitoring, and vicarious learning. The goal-setting process guided participants to set goals, and identify barriers and strategies to overcome them. Participants also self-monitored their own progress. Practising and mastering the behaviours sought to improve self-efficacy. The videos sought to engage participants in vicarious learning and also facilitate improvement in self-efficacy.

The Facebook component did not intend to incorporate all aspects of SCT, but rather complement the main intervention by providing feedback/reinforcement and social support 
(through the other participants and the facilitator), enhancing self-efficacy, addressing barriers, and providing further vicarious learning opportunities through the facilitated Facebook discussions.

Participants were encouraged to post information during each module. It was anticipated that members would gain the most benefit from actively engaging (by posting or commenting) based on findings of some previous research on online health discussion groups which has indicated that participants who write posts or comments asking questions or providing support to others achieve greater benefits than passive users (Mo \& Coulson, 2010; Oh \& Lee 2012; Tanis, Das \& Fortgens-Sillmann, 2011). Each cohort had a separate Facebook group to encourage discussion between group members which was relevant to the module being completed at any point in time.

There was one standard facilitator post per module reminding participants to log into the website to complete the current module and to encourage members to share ideas and experiences in relation to that module. During Module 1, participants were asked to introduce themselves to the group. Throughout the healthy eating modules ( 2 and 3 ) participants were asked to share modified recipes and photos of healthy meals and snacks that they had tried. During Module 4, participants were asked to share photos, ideas and experiences to increase physical activity. Participants were asked to share their personal ideas and experiences in relation to reducing screen-time and improving sleep in Modules 5 and 6 respectively.

Incentives were provided to encourage participants to post, with one participant per module in each cohort being selected (throughout modules 2-6) to receive a shopping gift card. The facilitator also provided encouraging comments and answered questions. Additional posts were provided depending on the needs and engagement of the group (e.g. reminders to post, posts in response to interest on specific topics, as well as seasonal and weather-specific posts - such as healthy Christmas snacks and encouraging discussion 
regarding sleep routines during daylight savings transition, and wet weather physical activity options). Posts and comments were monitored daily to ensure that the online discussion content was consistent with evidence-based guidelines.

\section{Measures}

Data were collected face-to-face at baseline and 3- and 6-months follow-up. A stadiometer was used to calculate height to the nearest $0.1 \mathrm{~cm}$ and a SECA scale was used to measure weight to the nearest $0.1 \mathrm{~kg}$. BMI was calculated using a standardized method (National Health and Medical Research Council, 2013). A third measurement was taken if there was a difference of more than $0.5 \mathrm{~cm}$ or $0.5 \mathrm{~kg}$ between the height and weight measurements respectively.

Parent-reported measures on demographics (baseline only), food intake, sleep, child feeding and role modelling were collected on an iPad which contained questionnaires previously assessed for validity and reliability where possible. Intake of fruit, vegetables and discretionary foods were assessed by a questionnaire (modified from the Eating and Physical Activity Questionnaire (Bennett, De Silva-Sanigorski, Nichols, Bell \& Swinburn, 2009). Discretionary food items included takeaway or fast food; sugary cereals; potato chips or other salty snacks, sweets, cakes, doughnuts, sweet biscuits or muffins; and sugary drinks. Responses to this question were used to determine a frequency of discretionary intake score. Parents also completed a 24-hour recall of their child's dietary intake (using the 'Easy Diet Diary’ app (Xyris Software (Australia) Pty Ltd)), which was used to determine $\mathrm{kJ} / \mathrm{kg}$ of body weight, percentage of $\mathrm{kJ}$ from saturated fat and percentage of $\mathrm{kJ}$ from sugar.

Physical activity was measured using Actigraph GT3X+ accelerometers (ActiGraph Corporation, Pensacola, FL). Children wore the monitors on an elasticized waist-belt for 7 days. Actilife version 6 (ActiGraph Corporation, Pensacola, FL) was used to analyse the data. The following cut-points were used to categorize activity; sedentary $<100$ counts/min, low 
light-intensity physical activity 101-800 counts/min, high light-intensity physical activity 801-1679 counts/min, moderate-intensity physical activity 1680-3367 count/min and vigorous-intensity physical activity $\geq 3368 \mathrm{count} / \mathrm{min}$ (Pate, Almeida, McIver, Pfeiffer \& Dowda, 2006).

The usual number of hours of screen-time per day on weekends and weekdays were determined using a parent-reported questionnaire (Downing, Hinkley \& Hesketh, 2015, Hinkley, Salmon, Okely, Crawford \& Hesketh, 2012)). Accelerometer data in conjunction with a parent-reported questionnaire (Sneddon, Peacock \& Crowley, 2013) were used to determine sleep duration and sleep latency. Parents reported on child feeding ('pressure to eat' and 'restriction' sub-scales) (Birch et al., 2001) via a set of questions on a five-point Likert scale.

Facebook group content was reviewed to determine the number of facilitator and participant posts, comments and 'likes' for each of the modules. Total active engagement for each participant was determined by tallying total comments and posts (aligning with the definition of 'active' engagement used in a similar study recently (Fiks et al., 2017)). Participants were also asked to complete a process evaluation questionnaire, which included one question about the usefulness of the Facebook component.

\section{Statistical analysis}

Differences in changes over time for total active Facebook engagement were assessed using linear mixed models. Baseline values and age were included as covariates. Intention-totreat (ITT) principles were used, regardless of whether participants attended all data collection time-points or completed the intervention. All tests were conducted in IBM SPSS Statistics for Windows, version 25 (IBM Corp., Armonk, N.Y., USA). 


\section{Results}

Enquiries were initially received from 372 parents/carers, who were provided with an information sheet. After reading the information sheet, 159 expressed an interest. After initial eligibility screening, 104 were deemed provisionally eligible. At baseline, where 93 parent/carer dyads attended, eligibility was confirmed for 86 participants. Forty-two participants were then randomly assigned to the intervention group. Details regarding baseline demographics, study flow, and participant retention have been published previously (Hammersley et al., 2019).

\section{Participant characteristics}

Thirty-six (86\%) participants from the intervention group joined a Facebook group. Although participants were asked at enrolment if they had a Facebook account or were willing to create one, there were 6 participants who did not join a group. Table 1 outlines the characteristics of participants invited to join a Facebook group. The number of participants per Facebook group ranged from three to ten. A considerable proportion of participants $(67 \%)$ 'liked', commented or posted on at least one module; participation in at least two modules was somewhat lower (50\%). Fewer participants 'liked', commented or posted in at least three modules $(38 \%)$.

\section{Facebook activity and engagement}

Table 2 displays the standard facilitator posts for each module, the percentage of participants who viewed the standard post and the mean (and SD) total number of facilitator posts for each module. Between $67 \%$ and $89 \%$ of participants viewed the standard facilitator posts, which varied depending on the module. Post viewing also differed according to cohort, e.g. an average of only $60 \%$ of participants viewed the standard facilitator posts in cohort 1 , compared to $100 \%$ in cohort 6 . There was a low level of engagement with the facilitator 
posts. There were only 10 comments in total to the standard facilitator posts throughout all cohorts. However, most activity related to the standard posts arose from participants creating in response to the facilitator posts and comments from other members on these participant posts.

Table 3 outlines the participant activity across all program modules. The total number of participant posts was 58 (average of 1.6 per group member), the total number of participant comments was 99 (average of 2.8 per group member) and the total number of likes was 135 (average of 3.8 per group member). There was a high degree of variation between participants, with two participants contributing seven posts each and 16 participants not posting or commenting at all. Posts also varied according to the cohort: generally larger cohorts had more posts and comments per member than smaller cohorts. Cohort 1, which was the largest (10 members), had an average of 2.8 posts per member. Cohort 5 , which was the smallest ( 3 members), had no participant posts at all. Module 2 (healthy meals) had the highest level of engagement and Module 3 (healthy snacks and drinks) had the lowest engagement.

The content of most participant posts and comments were largely in response to the standard facilitator post and therefore featured ideas and experiences in relation to the respective modules, including photos and recipes of healthy meals, photos and/or descriptions of healthy snacks, physical activities, screen-time alternatives and sleep routines. Some participant posts focused on clarification of some of the module content and other posts asked questions about website functionality. Some examples of participant posts are displayed in Table 4.

\section{Primary and secondary outcomes}

Table 5 displays the intention-to-treat analysis assessing differences over time by the level of active Facebook engagement. There was no significant effect for BMI. The linear 
mixed model analyses found a significant relation for sleep duration (estimate $1.79,95 \% \mathrm{CI}$ 0.42 to $3.17, \mathrm{p}=0.01)$ and there was also a significant relation for percentage of moderate- to vigorous-intensity physical activity (MVPA) in the non-hypothesized direction (estimate $0.14,95 \%$ CI -0.26 to $-0.01, \mathrm{p}=0.03)$. No other significant effects were found; however results for serves of vegetables (estimate $0.70,95 \%$ CI 0.54 to $0.00, p=0.06$ ) and child feeding - restriction (estimate $-0.02, \mathrm{CI}-0.05$ to $-0.05, \mathrm{p}=0.07$ ) were borderline in the hypothesized directions, and the result for light, moderate and vigorous physical activity (LMVPA) was borderline in the non-hypothesized direction (estimate -0.20 , CI -0.41 to 0.02, $\mathrm{p}=0.07)$.

\section{Process evaluation}

Thirty-seven intervention group participants (88\%) completed the process evaluation questionnaire. Despite a high level of user acceptability of the Time2bHealthy program overall, as previously reported (Hammersley et al., 2019), only 15 participants (41\%) agreed or strongly agreed that the Facebook component was useful.

\section{Discussion}

Parent-targeted health behaviour change interventions that incorporate social media have great potential, as parents are active users of social media sites (Duggan et al., 2015; Niela-Vilen et al., 2014). Surprisingly there have been few child health interventions involving parents which have included a social media component and very few of these have targeted childhood healthy lifestyle behaviours (Downing et al., 2017; Fiks et al., 2017; Gruver et al., 2016; Ling et al., 2018; Ruotsalainen et al., 2015; Swindle et al., 2018). The aim of this paper was to explore the level of participant engagement in the Facebook component of the Time2bHealthy program and to determine if the level of participant engagement influenced child health-related outcomes. This is one of the first studies to 
explore the effect of a parent-focused social media component of an online intervention on childhood obesity-related behaviors.

There was a high level of Facebook group membership and while the majority of participants posted, commented or 'liked' in at least one of the six modules, overall engagement was quite modest. These results are comparable with other similar studies that targeted childhood healthy lifestyle behaviors through parents (Downing et al., 2017, Swindle et al., 2018). Higher engagement has been demonstrated in other studies, but these have been solely social media-based (Fiks et al., 2017; Gruver et al., 2016), and therefore the demands placed on participants were probably not as high as other dual-component studies such as Time2bHealthy. Other similar studies have not provided detailed information on engagement (Ling et al., 2018; Ruotsalainen et al., 2015). Two recent systematic reviews which had a broader focus, investigating social media interventions targeting healthy lifestyle behaviors and behavior change in either children or adults, have reported a low level of engagement (Maher et al., 2014; Williams, Hamm, Shulhan, Vandermeer \& Hartling, 2014).

There was variation in the level of engagement across the different modules and between the different Facebook groups, which is also similar to other studies (Downing et al., 2017; Swindle et al., 2018). In groups with more members, there were generally more comments per member. It appears that there needs to be a minimum core group to elicit more engagement, highlighted by the smallest group of three participants that had no posts at all. Participation in the Facebook group was not compulsory, which may have had an influence on engagement. Participant acceptance was lower than expected, with less than half of participants reporting that it was useful. Similar studies have reported much higher rates of acceptance (Downing et al., 2017; Fiks et al., 2017; Ling et al., 2018); however two of these studies used Facebook as the sole intervention. 
Previous studies in health-related online communities have reported that there is generally a modest level of active participation, with almost half of participants being "lurkers", who read but rarely contribute posts or comments (Preece, Nonnecke \& Andrews, 2004). Reasons for a low level of active participation have been suggested, such as a lack of necessary skills, not being interested in the interactions, not fitting in, or not feeling that other members would benefit from their input (Preece et al., 2004), factors which could possibly have impacted on engagement in the current study.

Some studies have reported superior benefits for participants who actively engage by posting/commenting compared to those who participate passively (Mo \& Coulson, 2010; Oh \& Lee 2012; Tanis et al., 2011). It has been proposed that posters may benefit from the thought process involved in composing their post or comment to express their ideas, feelings and/or experiences (Ziebland \& Wyke, 2012). However, some studies have reported that lurkers also receive some benefit. For example, in a study of participants in health-related online support groups, there were enhanced benefits for some outcomes for participants who actively posted, while for measures of empowerment, results were similar for posters and lurkers (van Uden-Kraan, Drossaert, Taal, Seydel \& van de Laar, 2008).

Two studies investigating the effect of online breast cancer support had conflicting results. One study found that lurkers gained better benefits in regard to perceived well-being than participants who engaged more actively (Han, Hou,Kim, \& Gustafson, 2014)), whereas the other study found that active participation produced better results for some outcomes (Setoyama, Yamazaka \& Namayama, 2011). It is therefore possible that the participants in the Time2bHealthy study who viewed content without posting or commenting may have gained a benefit that we were unable to detect. Due to conflicting results of past studies on the possible benefits of "lurking", further research is clearly needed in this area. 
Time2bHealthy participants were generally unknown to each other. Had participants been familiar with each other, the engagement level may have been higher. For example, if they had been a member of the same playgroup, or if there had been face-to-face contact allowing members of the group to get to know each other, they may have been more willing to post and share information with the group. However, the evidence in the literature is divided on this topic. A physical activity Facebook intervention for new Mums, which used 'snowballing' recruitment by users inviting friends to join, reported a high level of engagement (Kernot, Olds, Lewis \& Maher, 2014). Therefore, recruiting existing friendship groups may be an avenue to explore to enhance engagement. However, familiarity with other Facebook group members did not result in a higher level of engagement in a nutrition and physical activity program for parents of infants which involved face-to-face sessions (Downing et al., 2017). Previous research has highlighted concerns regarding participant views on confidentiality of information posted (Moorhead et al., 2013) and some studies have indicated that participants may be more likely to share information if they are not known to other participants as they may feel that it is a less threatening environment to openly share information and seek help online (Haslam et al., 2017).

We found no significant difference in change in child BMI, dietary intake, child feeding or sedentary time according to Facebook active engagement level. Positive outcomes were demonstrated for parents who highly engaged in Facebook compared to those who had a lower engagement level in relation to sleep duration in the hypothesized direction. These findings may be related to the relatively high number of participant posts in this module. The sleep module elicited the second highest number of posts and it is possible that members of the group obtained more benefits through taking the time to consider and write about their own experiences and viewing other participants' experiences and ideas. The sleep module of the website contained only one video, whereas other modules contained at least three. 
Therefore, throughout the sleep module, the participants may not have obtained vicarious learning through the website content, but have gained benefit from the additional sharing of ideas and experiences of others in the Facebook group.

There was a significant change in MVPA according to engagement level in the nonhypothesized direction. The reason for this finding is unclear, but could possibly be due to an undesired effect of parents logging onto Facebook to access the group and then spending more time on social media than intended, and as a result of this, having less time to engage with their children in physical activity. As timed social media usage data was not collected, it is not possible to determine if could be the reason for this result. Other similar studies have also been unable to achieve improvements in physical activity (Downing et al., 2017; Ruotsalainen et al., 2015), with the exception of one feasibility study (Ling et al., 2018). Social media healthy lifestyle interventions in adults or children in general have also to date been unsuccessful in demonstrating positive changes in physical activity (Williams et al., 2014). This is an area of further investigation.

The modest results of this study align with many other general health-related social media studies that have also not demonstrated changes in key outcomes measured (Brindal et al., 2012; Maher et al., 2014; Taiminen, 2016; Williams et al., 2014). However, it is possible that other unmeasured benefits could have resulted from the intervention, such as participants' feeling of achievement, social support, and plans to implement healthy behaviors in the future. Therefore a longer follow-up period may be needed to demonstrate the impact of any such benefits on key outcomes (Taiminen, 2016) and it may be warranted for future studies to collect measures from participants on the immediate personal benefits they have gained.

The SCT constructs which were used in the Facebook component of this study aimed to complement and/or enhance those incorporated in the online modules. Specifically, 
vicarious learning was facilitated in the Facebook groups by general encouragement of participants to share ideas, experiences and photos in relation to the module content; feedback and reinforcement was provided by the facilitator in response to participant posts; and social support was addressed through general encouragement to comment on other participants' posts. However; the potential for these SCT constructs to impact on outcomes was likely limited due to the modest level of engagement in the Facebook groups.

Facilitator posts may have had more effect and engagement could have been enhanced if the posts were more targeted and specifically aligned with SCT constructs, rather than the more general approach that was used where most posts asked participants to share their ideas, experiences, or photos in relation to the particular topic. While it is difficult to make comparisons or recommendations based on similar studies, as a high level of detail is lacking, Facebook interventions which have used SCT in other populations may provide some insight. A recent successful weight maintenance study in adults specifically aligned facilitator posts with targeted SCT constructs such as self-control, vicarious learning, and behavioral capability (Hales, Davidson, \& Turner-McGrievy, 2014). A recent feasibility study of breast cancer survivors also successfully used SCT constructs, such as self-efficacy, reducing barriers, and enhancing social support, to formulate weekly tip Facebook posts (Pope, Lee, Zeng, Lee, \& Gao, 2019). Therefore; it is possible that the facilitator posts used in the current study may have been more effective had they been more specifically aligned with SCT constructs.

Careful consideration was given in regard to the type of social media platform employed for the current study. In the formative research for this study, a discussion board was used which was integrated into the web application (Jones, Wells, Okely, Lockyer \& Walton, 2011). This forum allowed for easy searching of posts through threads and an easy administration process as there was no need to add members to groups. However, while 
discussion forums are still being used in studies (Hamm et al., 2014), use has declined over recent years, and participants are probably not as familiar with their use as they would be of Facebook and other social media.

In a systematic review on social media use in child health, it was suggested that researchers harness technology platforms that people are already using (Hamm et al., 2014). Due to the surge in popularity of Facebook (Sensis, 2017) and the rising use of parenting groups on Facebook (Niela-Vilén et al., 2014), the use of this platform was explored. The high number of current users (Sensis, 2017), familiarity, ease of use, immediate access/notification of posts and accessibility on a variety of devices were important factors in selecting Facebook for use in this study. Some problems were encountered though that would not have been experienced with a discussion board. Several participants couldn't remember the email address that they signed up to Facebook with, which was needed to invite members to the group. Technical issues have also been reported in previous studies (Eysenbach, Powell, Englesakis, Rizo \& Stern, 2004; Welch et al., 2018). The way in which posts appear in the group may have been difficult for participants to navigate. It was not always the most recent posts that appeared first. Although links to the Facebook group were embedded in the web application, the additional step required to access the group may also have been a barrier to engagement. Another possible disadvantage of using a Facebook group which has been documented, is the spread of misinformation (Welch et al., 2018; Westberg, Stavros, Smith, Munro \& Argus, 2018). This risk was negated in this study as the groups were monitored by the facilitator to ensure that any information that was discussed was consistent with evidencebased guidelines. Past research has highlighted the advantages of peer-support and also stressed the importance of a professional facilitator being involved (Niela-Vilén et al., 2014). Further research is suggested to investigate the most appropriate social media platform to be utilised for parent-focused childhood healthy lifestyle interventions, which is difficult given 
the rapidly changing landscape in this space. It is important for researchers to keep abreast with current trends and explore the feasibility of popular platforms for use in interventions.

Facebook and other social media sites allow users to access information and engage with others at a time that is convenient to them. The Time2bHealthy Facebook component therefore offered parents the flexibility of accessing it at a time which suited their schedule, enabling them to work around children's sleep and activity times.

The significant findings of this Facebook analysis are different to the significant outcomes reported in the main outcomes of the study, indicating that engagement in the Facebook component of the program had a unique effect on outcomes, which varied from the effect of the overall program. No significant differences in BMI change between participants were found based on the level of active engagement in the Facebook group. Our null BMI outcome is consistent with the outcome of the main study and is possibly due to the large proportion of children in the healthy weight range and the underpowered sample size, as previously discussed (Hammersley et al., 2019).

\section{Strengths and Limitations}

This study is one of the first to explore the effect of parent engagement in a social media component of a childhood healthy lifestyle intervention and therefore makes a valuable contribution to the literature. There are a number of limitations. The Facebook groups were used at a very basic level. Additional functionality such as polls, scheduled posts, events, uploading documents were not used. Process evaluation of the Facebook group was limited and further qualitative research on what aspects of the Facebook group were useful for participants would be useful. The analysis conducted was based on participants' 'active' engagement which was defined as comments and posts. Therefore, it was not determined from the analysis if participants who did not actively engage by commenting or posting were gaining a benefit by just 'liking' or viewing the posts and comments of others. We also did 
not collect information on the amount of time participants spent interacting with the Facebook group. Even though posts were 'liked' or 'seen', it is therefore difficult to determine the extent to which the participants read and considered the content.

\section{Conclusion}

This is one of the first parent-focused childhood healthy lifestyle interventions which has used a Facebook component. The results of this study indicated that higher active engagement in a Facebook component to an online healthy lifestyle program for parents of preschool-aged children did not result in superior changes in BMI compared to participants with low engagement but did result in positive changes in sleep duration. There was modest user acceptability of the Facebook group and the majority of participants joined and engaged on at least one instance with the group. More research is warranted with larger sample sizes and longer duration to further explore the potential of social media in parent-focused childhood healthy lifestyle interventions. 


\section{References}

Bandura, A. (1989). Social Cognitive Theory. In: VASTA, R. (ed.) Annals of child development Volume 6. Six Theories of Child Development. Greenwich, CT: JAI Press.

Bennett, C. A., De Silva-Sanigorski, A. M., Nichols, M., Bell, A. C., \& Swinburn, B. A. (2009). Assessing the intake of obesity-related foods and beverages in young children: comparison of a simple population survey with $24 \mathrm{hr}-$ recall. International Journal of Behavioral Nutrition and Physical Activity, 6, 71. doi:10.1186/1479-5868-6-71

Birch, L. L., Fisher, J. O., Grimm-Thomas, K., Markey, C. N., Sawyer, R., \& Johnson, S. L. (2001). Confirmatory factor analysis of the Child Feeding Questionnaire: a measure of parental attitudes, beliefs and practices about child feeding and obesity proneness. Appetite, 36, 201-10. doi:10.1006/appe.2001.0398

Brindal, E., Freyne, J., Saunders, I., Berkovsky, S., Smith, G., \& Noakes, M. (2012). Features predicting weight loss in overweight or obese participants in a web-based intervention: Randomized trial. Journal of Medical Internet Research, 14. doi:10.2196/jmir.2156

Chen, J. L., Weiss, S., Heyman, M. B., Cooper, B., \& Lustig, R. H. (2011). The efficacy of the web-based childhood obesity prevention program in Chinese American adolescents (Web ABC study). Journal of Adolescent Health, 49, 148-54. doi:10.1016/j.jadohealth.2010.11.243

De Bruyn, A., \& Lilien, G. L. (2008). A multi-stage model of word-of-mouth influence through viral marketing. International Journal of Research in Marketing, 25, 151163. doi: https://doi.org/10.1016/j.ijresmar.2008.03.004

Doub, A. E., Small, M., \& Birch, L. L. (2016). A call for research exploring social media influences on mothers' child feeding practices and childhood obesity risk. Appetite, 99, 298-305. doi:10.1016/j.appet.2016.01.003 
Downing, K. L., Campbell, K. J., van der Pligt, P., \& Hesketh, K. D. (2017).

Facilitator and participant use of Facebook in a community-based intervention for parents: the InFANT extend program. Childhood Obesity, 13, 443-454. doi:10.1089/chi.2017.0078

Downing, K. L., Hinkley, T., \& Hesketh, K. D. (2015). Associations of parental rules and socioeconomic position with preschool children's sedentary behaviour and screen time. Journal of Physical Activity and Health, 12, 515-21. doi:10.1123/jpah.2013-0427

Duggan, M., Lenhart, A., Lampe, C., \& Ellison, N. (2015). Parents and Social Media. Retrieved from http://www.pewinternet.org/2015/07/16/parents-and-social-media/

Eysenbach, G., Powell, J., Englesakis, M., Rizo, C., \& Stern, A. (2004). Health related virtual communities and electronic support groups: systematic review of the effects of online peer to peer interactions. British Medical Journal, 328, 1166. doi:10.1136/bmj.328.7449.1166

Fiks, A. G., Gruver, R. S., Bishop-Gilyard, C. T., Shults, J., Virudachalam, S., Suh, A. W. . Power, T. J. (2017). A social media peer group for mothers to prevent obesity from infancy: The Grow2Gether randomized trial. Childhood Obesity, 13, 356-368. doi:10.1089/chi.2017.0042

Golley, R. K., Hendrie, G. A., Slater, A., \& Corsini, N. (2011). Interventions that involve parents to improve children's weight-related nutrition intake and activity patterns what nutrition and activity targets and behaviour change techniques are associated with intervention effectiveness? Obesity Reviews, 12, 114-130. doi:10.1111/j.1467789X.2010.00745.X

Gruver, R. S., Bishop-Gilyard, C. T., Lieberman, A., Gerdes, M., Virudachalam, S., Suh, A. W. . Fiks, A. G. (2016). A social media peer group intervention for mothers to prevent obesity and promote healthy growth from infancy: development and pilot trial. JMIR Research Protocols, 5, e159. doi: 10.2196/resprot.5276 
Hales, Z., Davidson, C., \& McGrievy. (2014). Varying social media post types differentially impacts engagement in a behavioral weight loss intervention. Translational Behavioural Medicine, 4, 355-362. Doi: 10.1007/s13142-014-0274-Z

Hamm, M. P., Shulhan, J., Williams, G., Milne, A., Scott, S. D., \& Hartling, L. (2014). A systematic review of the use and effectiveness of social media in child health. BMC Pediatrics, 14. doi:10.1186/1471-2431-14-138

Hammersley, M. L., Okely, A. D., Batterham, M. J., \& Jones, R. A. (2019). An internet-based childhood obesity prevention program (Time2bHealthy) for parents of preschool-aged children: Outcomes of a randomized controlled trial. Journal of Medical Internet Research, 21, e11964. doi:10.2196/11964. .

Hammersley, M. L., Jones, R. A., \& Okely, A. D. (2017). Time2bHealthy - An online childhood obesity prevention program for preschool-aged children: A randomised controlled trial protocol. Contemporary Clinical Trials, 61, 73-80. doi:10.1016/j.cct.2017.07.022

Han, J.Y., Hou, J., Kim, E., \& Gustafson, D.H. (2014). Lurking as an active participation process: a longitudinal investigation of engagement with an online support group. Health Communication, 29, 911-923. doi: 10.1080/10410236.2013.816911

Haslam, D. M., Tee, A., \& Baker, S. (2017). The use of social media as a mechanism of social support in parents. Journal of Child and Family Studies, 26, 2026-2037. doi:10.1007/s10826-017-0716-6

Hinkley, T., Salmon, J., Okely, A. D., Crawford, D., \& Hesketh, K. (2012). The HAPPY study: development and reliability of a parent survey to assess correlates of preschool children's physical activity. Journal of Science and Medicine in Sport, 15, 407-17. doi:10.1016/j.jsams.2011.12.009 
Jones, R., Wells, M., Okely, A., Lockyer, L. \& Walton, K. (2011). Is an online healthy lifestyles program acceptable for parents of preschool children? Nutrition \& Dietetics, 68, 149-154.

Kernot, J., Olds, T., Lewis, L. K. \& Maher, C. (2014). Usability testing and piloting of the Mums Step it up Program - a team-based social networking physical activity intervention for women with young children. PLOS ONE, 9, e108842. doi: 10.1371/journal.pone. 0108842

Ling, J., Robbins, L. B., Zhang, N., Kerver, J. M., Lyons, H., Wieber, N. \& Zhang, M. (2018). Using Facebook in a healthy lifestyle intervention: feasibility and preliminary efficacy. Western Journal of Nursing Research, 193945918756870. doi: $10.1177 / 0193945918756870$

Maher, C.A., Lewis., L.K., Ferrar, K., Marshall, S., De Bourdeauduij, I. \& Vandelanotte, C. (2014). Are health behavior change interventions that use online social networks effective? A systematic review. Journal of Medical Internet Research, 16, 1-17. doi: 10.2196/jmir.2952

Miller, M. A., Kruisbrink, M., Wallace, J., Ji, C., \& Cappuccio, F. P. (2018). Sleep duration and incidence of obesity in infants, children, and adolescents: a systematic review and meta-analysis of prospective studies. Sleep, 41. doi: 10.1093/sleep/zsy018

Mo, P., \& Coulson, N.S. (2010). Empowering processes in online support groups among people living with HIV/AIDS: A comparitive analysis of 'lurkers' and 'posters'. Computers in Human Behavior, 26, 1183-1193. doi: 10.1016/j.chb.2010.03.028

Moorhead, S. A., Hazlett, D. E., Harrison, L., Carroll, J. K., Irwin, A. \& Hoving, C. (2013). A new dimension of health care: systematic review of the uses, benefits, and limitations of social media for health communication. Journal of Medical Internet Research, 15, e85. doi: 10.2196/jmir.1933 
Natale, R. A., Messiah, S. E., Asfour, L., Uhlhorn, S. B., Delamater, A., \& Arheart, K. L. (2014). Role modeling as an early childhood obesity prevention strategy: effect of parents and teachers on preschool children's healthy lifestyle habits. Journal of Developmental and Behavioral Pediatrics, 35, 378-387. doi:

$10.1097 / \mathrm{dbp} .0000000000000074$

National Health and Medical Research Council. (2013). Clinical Practice Guidelines for the Management of Overweight and Obesity in Adults, Adolescents and Children. Melbourne.

Niela-Vilén, H., Axelin, A., Salanterä, S. \& Melender, H. L. (2014). Internet-based peer support for parents: a systematic integrative review. International Journal of Nursing Studies, 51, 1524-1537. doi: 10.1016/j.ijnurstu.2014.06.009

Oh, H.J., Lee, B. (2011). The effect of computer-mediated social support in online communities on patient empowerment and doctor-patient communication. Health Communication, 27, 30-41. doi: 10.1080/10410236.2011.567449

O'Kane, C., Wallace, A., Wilson, L., Annis, A., Ma, D. W. L. \& Haines, J. (2018). Family-based obesity prevention: perceptions of canadian parents of preschool-age children. Canadian Journal of Dietetic Practice and Research, 79, 13-17. doi: 10.3148/cjdpr-2017-027

Okely, A. D., \& Jones, R. A. (2011). Sedentary Behavior Recommendations for Early Childhood. In R. Tremblay, Barr, R., Peters, R. \& Boivin, M. (Ed.), Encyclopedia on Early Childhood Development Montreal: Centre for Excellence for Early Childhood Development. Okely, A. D., Salmon, J., Vella, S. A., Cliff, D., Timperio, A., Tremblay, M., . . . Marino, N. (2012). A Systematic Review to inform the Australian Sedentary Behaviour Guidelines for Children and Young People. Report prepared for the Australian Government Department of Health, June 2012. Canberra, Australia: Commonwealth of Australia. 
Pate, R. R., Almeida, M. J., McIver, K. L., Pfeiffer, K. A. \& Dowda, M. (2006). Validation and calibration of an accelerometer in preschool children. Obesity (Silver Spring), 14, 2000-6. doi: 10.1038/oby.2006.234

Pope, Z., Lee, J.E., Zeng, N., Lee, H.Y., \& Gao, Z. (2019). Feasibility of smartphone application and social media intervention on breast cancer survivors' health outcomes. Translational Behavioral Medicine, 9, 11-22. doi: 10.1093/tbm/iby002

Preece, J., Nonnecke, B. \& Andrews, D. (2004). The top five reasons for lurking: improving community experiences for everyone. Computers in Human Behavior, 20, 201223. doi: 10.1016/j.chb.2003.10.015

Ruotsalainen, H., Kyngas, H., Tammelin, T., Heikkinen, H. \& Kaariainen, M. (2015). Effectiveness of Facebook-delivered lifestyle counselling and physical activity selfmonitoring on physical activity and body mass index in overweight and obese adolescents: a randomized controlled trial. Nursing Research and Practice, 159205. doi: $10.1155 / 2015 / 159205$

Sensis (2017). Sensis Social Media Report 2017. Melbourne: Sensis. Retrieved from: https://irp-cdn.multiscreensite.com/535ef142/files/uploaded/Sensis-Social-Media-Report2017.pdf

Setoyama, Y., Yamazaki, Y., \& Namayama, K. (2011). Benefits of peer support in online Japanese breast cancer communities: Differences between lurkers and posters. Journal of Medical Internet Research, 13, e122. doi: 10.2196/jmir.1696

Smart Insights (2018). Global Social Media Research Summary. West Yorkshire: Smart Insights. Retrieved from https://www.smartinsights.com/social-mediamarketing/social-media-strategy/new-global-social-media-research/ 
Sneddon, P., Peacock, G. G. \& Crowley, S. L. (2013). Assessment of sleep problems in preschool aged children: an adaptation of the children's sleep habits questionnaire. Behavioral Sleep Medicine, 11, 283-96. doi: 10.1080/15402002.2012.707158

Strange, C., Fisher, C., Howat, P. \& Wood, L. (2018). 'Easier to isolate yourself...there's no need to leave the house' - A qualitative study on the paradoxes of online communication for parents with young children. Computers in Human Behavior, 83, 168175. doi: https://doi.org/10.1016/j.chb.2018.01.040

Swindle, T. M., Ward, W. L. \& Whiteside-Mansell, L. (2018). Facebook: the use of social media to engage parents in a preschool obesity prevention curriculum. Journal of Nutrition Education \& Behavior, 50, 4-10.e1. doi: 10.1016/j.jneb.2017.05.344

Taiminen, H. (2016). How do online communities matter? Comparison between active and non-active participants in an online behavioral weight loss program. Computers in Himan Behavior, 63, 787-795. doi: 10.1016/j.chb.2016.06.002

Tanis, M., Das, E., Fortgens-Sillman, M. (2011). Finding care for the caregiver? Active participation in online health forums attenuates the negative effect on caregiver strain on wellbeing. Communications, 36, 51-66. doi: 10.1515/comm.2011.003

Thackeray, R., Neiger, B. L., Hanson, C. L. \& McKenzie, J. F. (2008). Enhancing promotional strategies within social marketing programs: use of Web 2.0 social media. Health Promotion Practice, 9, 338-43. doi: 10.1177/1524839908325335

van Uden-Kraan, C.F., Drossaert, C.H., Taal, E., Seydel, E.R. \& van de Laar, M.A. (2008). Self-reported differences in empowerment between lurkers and posters in online parient support groups. Journal of Medical Internet Research, 10, e18. doi: 10.2196/jmir.992

Welch, V., Petkovic, J., Simeon, R., Presseau, J., Gagnon, D., Hossain, A., . . . Desmeules, M. 2018. Interactive social media interventions for health behaviour change, 
health outcomes, and health equity in the adult population. Cochrane Database of Systematic Reviews. doi: 10.1002/14651858.CD012932

Westberg, K., Stavros, C., Smith, A. C. T., Munro, G. \& Argus, K. (2018). An examination of how alcohol brands use sport to engage consumers on social media. Drug and Alcohol Review, 37, 28-35. doi: 10.1111/dar.12493

Williams, G., Hamm., M.P., Shulhan., J., Vandermeer, B., Hartling, L (2014). Social media interventions for diet and exercise behaviors: A systematic review and meta-analysis of randomized controlled trials. BMJ Open, 4, 1-16. doi: 10.1136/bmjopen-2013-003926

Williamson, D. A., Martin, P. D., White, M. A., Newton, R., Walden, H., YorkCrowe, E., .. . Ryan, D. (2005). Efficacy of an internet-based behavioral weight loss program for overweight adolescent African-American girls. Eating and Weight Disorders, 10, 193-203.

World Health Organization. (2012). Population-based approaches to Childhood Obesity Prevention. Geneva: World Health Organization.

Ziebland, S. \& Wyke, S. (2012). Health and illness in a connected world: How might sharing experiences on the Internet affect people's health? The Millbank Quarterly: A Multidiciplinary Journal of Population Health and Health Policy, 90, 219-249. 
Table 1

Baseline characteristics of participants whom were invited to join a Facebook group $(n=42)$

Characteristic

Number

Child sex

Boy $(\%)$

$24(57 \%)$

$\operatorname{Girl}(\%)$

$18(43 \%)$

Mean child age (years) (SD)

$3.360(0.804)$

Mean child BMI (SD)

$16.724(0.915)$

Child weight status
Healthy Weight (\%)
$40(95 \%)$
Overweight (\%)
$2(5 \%)$
Obese $(\%)$
$0(0 \%)$

Participating parent sex
Male $(\%)$
$2(5 \%)$
Female $(\%)$
$40(95 \%)$

Mean age participating parent (SD)

Highest level of education of participating parent
Not university qualified (\%)
$28(19 \%)$
University qualified (\%)
$32(76 \%)$
Currently studying (\%)
$2(5 \%)$

Participating parent income after tax

$<\$ 580 /$ week $(\%)$

$20(48 \%)$ 
\$580-\$1240/week (\%)

$>\$ 1240 /$ week $(\%)$

Mean BMI participating parent (SD)

Weight status participating parent
Underweight (\%)
Healthy Weight (\%)
Overweight (\%)
Obese $(\%)$
$2(5 \%)$
$25(60 \%)$
$9(21 \%)$
$6(14 \%)$

Marital status participating parent

Single/divorced/separated/widowed (\%)

Married/with partner (\%)
$16(38 \%)$

$6(14 \%)$

$24.810(4.636)$

$3(7 \%)$

$39(93 \%)$ 
Table 2

Facilitator posts

$\begin{array}{lll}\text { Module } & \text { Standard facilitator post } & \text { Mean } \\ \text { participants } & \text { number } \\ \text { that viewed } & \text { (SD) of } \\ & \text { standard } & \text { facilitator } \\ \text { facilitator } & \text { posts per } \\ & \text { post } & \text { module }\end{array}$

$1 \quad$ "Welcome to Time2bHealthy everyone! Please feel $\quad 80 \% \quad 1.000$

free to introduce yourself, post tips and ideas that you

would like to share and issues that you would like to

discuss with others. The idea of this Facebook group

is to discuss and share ideas and experiences on each

of the topics as we move through the Time2bHealthy

program. Sometimes the best advice can come from

other parents who are in a similar situation as you".

2 "Hi everyone, I hope you are enjoying module 2 and

$89 \%$

that you are finding the discussion on the Facebook

page useful. Just a reminder that it would be great to

share a recipe that you have modified to increase the amount of vegetables. A photo would also be great.

The more recipes, the better! We are offering a $\$ 20$

shopping gift card for the best modified recipe of the week". 
3 "Hi everyone, with module 3 now open, over the next $86 \%$

couple of weeks it would be great if you could take a

photo and share your favourite healthy snack with

others in the group. We are offering a \$20 shopping

gift card for one of our participants".

$4 \quad$ "As we turn our focus to physical activity in module $67 \%$

4, please remember to share any tips and ideas that

you have. Do you have any equipment that you find

helps to keep your family active? One post will be

selected to receive a $\$ 20$ shopping gift card at the end

of this module".

5 "As we turn to screen time in module 5, please

$78 \%$

remember to share any tips and ideas that you have.

One post will be selected to receive \$20 shopping gift

card at the end of this module".

6

"As we start the final module on sleep, please

$83 \%$

remember to share any experiences, tips and ideas

that you have. One post will be selected to receive s

\$20 shopping gift card at the end of this module". 
Table 3

Participant Facebook activity across all modules

\begin{tabular}{|c|c|c|c|c|c|c|c|}
\hline & Module 1 & Module 2 & Module 3 & Module 4 & Module 5 & Module 6 & Total \\
\hline 'Likes' & 4 & 31 & 19 & 38 & 28 & 15 & 135 \\
\hline Comments & 8 & 26 & 4 & 25 & 23 & 13 & 99 \\
\hline Posts & 1 & 20 & 9 & 11 & 11 & 18 & 58 \\
\hline Total & 13 & 77 & 32 & 74 & 62 & 46 & 292 \\
\hline
\end{tabular}

Table 4

Participant post examples

Module Participant post example

1 "Hi, I'm a mum of two children aged three and one. I'm looking forward to learning some new healthy lifestyle and eating strategies for the family. At the moment, any motivation to improve our eating habits is welcome".

2 "One of my current favourites for sneaking veg in is a baked meatballs recipe I found online, served with carrot and zucchini 'spaghetti'. It has spinach and potato hidden in the meatballs and both kids gobble it down".

3 "One of my daughter's favourite healthy snacks has been the beans we grew in the garden. These were the last of them, unfortunately, and she kept asking me if she could eat them while I was making tea. She hasn't been that keen on beans before. Not sure if they are sweeter when they are eaten fresh, or if she just 
enjoyed picking them. Although she wouldn't eat the cherry tomatoes she picked".

4 "My son love love loves swimming. This is him trying to catch a ball. Our local pool is indoor so we can go all year round regardless of the weather".

5 "Some of the strategies I have put in place this week to reduce our screen time (I needed to drop $1 / 2$ an hour to 1.5 hours a day roughly) have been:

- Get my pre-schooler involved in cooking dinner with me and I have set up the play kitchen next to my kitchen so my one year old "cooks/goes shopping" when I am cooking"

- Organised the card games/ puzzles/ dominos and have been getting these out for quiet time while my one year old has her nap.

- Starting going back to the local library to get some new books into the household to encourage more reading.

- My 3 year old started an "about me" folder which includes cutting and pasting pictures which he relates to (photos, favourite foods, activities, stickers etc) into the folder which he can show to people and talk about.

- Making sure I put away the different types of toys and bring them out separately so they stay engaged/ play with a variety of things and the house doesn't get completely trashed!

So far we are doing ok but it is very tempting to put the TV on so I can do tasks around the house by myself. Anyone else come up with some good strategies to reduce screen time?"

6 "Some sharing on bedtime routine

- We teach our girl how to read the clock. So when the set bedtime comes, she 
looks at the clock and knows it's the time to go to bedroom. Less arguments occur.

- When she was younger we set the alarm clock and when it ringed, she understood bedtime started now.

- We put a small bookshelf in her bedroom and keep the books only being read during bedtime. Somehow this makes her looking forward to bedtime so she can hears those stories.

- Set the rule that how many stories you are going to read. For us we only read one. If no rules she will ask one story after another. Make her more awake or overtired.

- Get changed in pyjamas also makes her know sleep time is about to come especially for younger kids. I will involve her when buying pyjamas. She's happy to be in beautiful pyjamas too.

- Our bedtime routine: Say goodnight to everyone in the house -> bath (if not done so before dinner) -> brush teeth $->$ get changed in pyjamas- $>$ read a story chosen by her $->$ sing a song $->$ cuddle and kiss $->$ turn night light on $->$ she sleeps on bed and I leave her room”. 
Table 5

Intention-to-treat analyses based on level of active engagement (total comments and posts) in Facebook group over time

\begin{tabular}{|c|c|c|c|c|}
\hline \multirow[t]{2}{*}{ Variable } & \multirow[t]{2}{*}{ Estimate } & \multicolumn{2}{|c|}{ 95\% Confidence Interval } & \multirow[t]{2}{*}{ P-value } \\
\hline & & Lower Bound & Upper Bound & \\
\hline BMI & 0.01 & -0.16 & 0.03 & 0.54 \\
\hline $\mathrm{kJ} / \mathrm{KG}$ of body weight ${ }^{\mathrm{b}}$ & 0.17 & -0.16 & 6.51 & 0.68 \\
\hline Percentage of kJ from sugar ${ }^{b}$ & 0.03 & -0.23 & 0.29 & 0.80 \\
\hline Percentage of $\mathrm{kJ}$ from saturated fat ${ }^{\mathrm{b}}$ & 0.27 & -0.14 & 0.28 & 0.20 \\
\hline Serves of fruit ${ }^{\mathrm{c}}$ & 0.28 & -0.01 & 0.01 & 0.16 \\
\hline Serves of vegetables ${ }^{c}$ & 0.71 & 0.54 & 0.00 & 0.06 \\
\hline Discretionary food frequency score & 0.02 & -0.09 & 0.14 & 0.66 \\
\hline Child feeding - Restriction ${ }^{e}$ & -0.02 & -0.05 & 0.00 & 0.07 \\
\hline Child feeding - Pressure ${ }^{\mathrm{e}}$ & 0.70 & 0.46 & 0.01 & 0.11 \\
\hline Sleep duration (hours) ${ }^{\mathrm{f}}$ & 1.79 & 0.42 & 3.17 & 0.01 \\
\hline Sleep reluctances & 0.22 & -0.02 & 0.07 & 0.34 \\
\hline Percentage sedentary time & 0.06 & -0.14 & 0.27 & 0.55 \\
\hline
\end{tabular}




\section{Percentage LMVPA ${ }^{\circ}$}

Percentage MVPA
$-0.20$

$-0.14$
$-0.41$

$-0.26$
0.02

$-0.01$
0.07

0.03

Note. ${ }^{a} \mathrm{n}=42$ - Linear mixed model (random intercept, variance components). Estimate indicates result for primary outcome. Age, cohort and baseline values included as covariates in the model. Significant at $\mathrm{p}<0.05$; ${ }^{\mathrm{b}}$ Calculated from 24-hour diet recall using Easy Diet Diary/Foodworks; ${ }^{\mathrm{c}}$ From Food Questionnaire, ${ }^{\mathrm{d}}$ Scored from food questionnaire questions on frequency of intake of takeaway or fast food; sugary cereals; potato chips or other salty foods; sweets; and cakes doughnuts, sweet cookies or muffins. Responses of never or rarely; 1-3 times per month; 1-2 times per week; 3-4 times per week; 5-6 times per week; once per day; and 2 or more times per day were coded as 1-6 respectively and summed to obtain a discretionary food score; ${ }^{\mathrm{e}}$ Child feeding questionnaire; ${ }^{\mathrm{f}}$ Accelerometer-measures ${ }^{\mathrm{g}}$ From sleep questionnaire. 